\section{(6) OPEN ACCESS}

\title{
In vivo confocal microscopy and histopathology of the conjunctiva in trachomatous scarring and normal tissue: a systematic comparison
}

\author{
Victor $\mathrm{H} \mathrm{Hu},{ }^{1}$ Martin J Holland, ${ }^{2}$ lan A Cree, ${ }^{3}$ James Pullin, ${ }^{3}$ Helen A Weiss, ${ }^{4}$ \\ Patrick Massae, ${ }^{5}$ William Makupa, ${ }^{5}$ David C W Mabey, ${ }^{2}$ Robin L Bailey, ${ }^{2}$ \\ Matthew J Burton, ${ }^{1}$ Phil Luthert $^{3}$
}

- Additional material is published online only. To view please visit the journal online (http://dx.doi.org/10.1136/ bjophthalmol-2013-303126).

${ }^{1}$ Faculty of Infectious and Tropical Diseases, International Centre for Eye Health, London School of Hygiene and Tropical Medicine, London, UK

${ }^{2}$ Department of Clinical Research, Faculty of Infectious and Tropical Diseases, London School of Hygiene and Tropical Medicine, London, UK ${ }^{3}$ Institute of Ophthalmology, University College London, London, UK

${ }^{4}$ Department of Infectious Disease Epidemiology, Faculty of Epidemiology and

Population Health, London School of Hygiene and Tropical Medicine, London, UK

${ }^{5}$ Eye Department, Kilimanjaro Christian Medical Centre, Moshi, Tanzania

Correspondence to Victor Hu, Faculty of Infectious and Tropical Diseases, International Centre for Eye Health, London School of Hygiene and Tropical Medicine, Keppel Street, London WC1E 7HT, UK;

victor.hu@|shtm.ac.uk

Received 15 January 2013

Revised 27 May 2013

Accepted 29 June 2013

Published Online First

6 August 2013

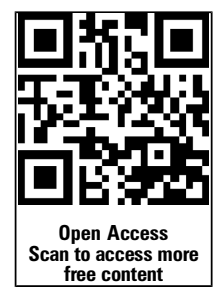

\footnotetext{
To cite: $\mathrm{Hu} \mathrm{VH}_{\text {, }}$

Holland MJ, Cree IA, et al. Br J Ophthalmol

2013;97:1333-1337.
}

\section{ABSTRACT}

Aim To compare in vivo confocal microscopy (IVCM) with the histopathological examination of tissue and cellular changes in normal and diseased conjunctiva. Methods Participants underwent clinical examination and IVCM of the tarsal conjunctiva. A biopsy of the upper tarsal conjunctiva was collected and stained with tinctorial stains and by immunohistochemical staining for CD45 and CD83. Connective tissue scarring, inflammatory cell density and the presence of dendritiform cells were quantitatively assessed in a masked manner by both IVCM and histological assessments for comparative analysis.

Results Thirty-four participants with severe trachomatous conjunctival scarring and 33 participants with healthy conjunctiva were recruited. The IVCM connective tissue scarring score was strongly associated with the histological grading of scarring $(p<0.001)$. There was limited evidence of an association between the IVCM inflammatory cell infiltrate and the histological inflammatory cell grade $(p=0.05)$. We did not find any evidence to support the hypothesis that dendritiform cells seen with IVCM are mature, conventional dendritic cells.

Conclusions The results show that IVCM can be used to robustly quantitate connective tissue scarring and also has a role in measuring the inflammatory cell infiltrate. The discordance between IVCM dendritiform cells and immunohistochemical dendritic cells may be a result of study limitations or may be because these dendritiform structures represent another cell type, such as fibroblasts, rather than dendritic cells.

\section{INTRODUCTION}

In vivo confocal microscopy (IVCM) provides highresolution images of living tissues in a non-invasive manner. It relies on the optical principle of confocality of the illumination and observation systems, that is, the two systems are focused on the same focal points. ${ }^{1}$ Light outside of the focal plane is highly suppressed, thereby markedly increasing the resolution. This is in contrast with light biomicroscopy, for example, with a slit lamp, where there is a large amount of scattered light that limits the maximum resolution which can be attained. The confocal field of view is very small and so the microscope rapidly scans the focal point across the tissue and reconstructs an image. The image obtained is parallel to the surface being examined.
IVCM is used in several medical disciplines, including dermatology and gastroenterology, where it has been used to examine for malignant changes. ${ }^{2}{ }^{3}$ In ophthalmology, its uses include the diagnosis and monitoring of microbial keratitis, where it may help to identify acanthamoeba cysts and filamentary fungi; assessment of potentially malignant lesions; and examination of the ocular surface after glaucoma and other forms of surgery. ${ }^{4}$

Trachoma is a chronic, scarring keratoconjunctivitis initiated by recurrent infection with Chlamydia trachomatis in childhood. The scarring complications of the disease, usually seen in adulthood, include tarsal conjunctival scarring, trichiasis and corneal opacity. We have previously used IVCM to quantify both inflammatory and scarring changes in the tarsal conjunctiva in cicatricial trachoma. $^{5}{ }^{6}$ However, interpreting IVCM images can be challenging and this is especially true for IVCM of the conjunctiva, underlying tarsal/bulbar tissue and lid margin. These contain many different structures and cell types, including goblet cells, Meibomian glands, lymphoid tissue and pseudoglands of Henle.

To validate the interpretation of IVCM images of the tarsal conjunctiva, including those from our previous studies on trachomatous scarring, we systematically compared IVCM with histological/ immunohistochemical examination of the tarsal conjunctival surface. Participants with trachomatous conjunctival scarring (many of whom also had marked inflammation) and participants with healthy conjunctiva were recruited. We examined the hypotheses that the IVCM scarring score and inflammatory cell count would correspond to the grading of histological parameters, and that IVCM dendritiform cells (DFC) would be associated with the immunohistological evidence of dendritic cells (DC). Previously, there have been some attempts to compare IVCM and histological findings, but these are limited to qualitative comparisons and have employed impression cytology specimens, which do not sample subepithelial tissue. ${ }^{78}$

\section{MATERIALS AND METHODS \\ Subjects}

This study adhered to the tenets of the Declaration of Helsinki. It was approved by the Tanzanian National Institute of Medical Research Ethics Committee, the Kilimanjaro Christian Medical Centre Ethics Committee and the London School 
of Hygiene and Tropical Medicine Ethics Committee. The study was explained to the potential study cases, and written, informed consent was obtained before enrolment. Thirty-four individuals with trachomatous trichiasis were recruited from those undergoing trichiasis surgery in the Kilimanjaro region. Thirty-three comparison participants with healthy tarsal conjunctiva were recruited from patients undergoing cataract or retinal detachment surgery at Kilimanjaro Christian Medical Centre.

\section{Clinical examination}

All individuals were examined by an ophthalmologist using $\times 2.5$ loupes and a bright torch. The 1981 WHO (FPC) trachoma grading system was used to grade conjunctival follicles and papillae, entropion, trichiasis and corneal opacity. ${ }^{9}$ A more detailed grading system was used for conjunctival scarring as previously described. ${ }^{6} 10$

\section{Confocal microscopy assessment}

IVCM examination of the upper tarsal conjunctiva was performed using the Heidelberg Retina Tomograph 3 (HRT3) in combination with the Rostock Corneal Module (Heidelberg Engineering $\mathrm{GmbH}$, Dossenheim, Germany). Image grading protocols, as previously described, were used to grade features, including the inflammatory cell density, the presence of DFC and subepithelial connective tissue scarring. These protocols are shown shown in online supplementary figures S1 and S2. ${ }^{5} 611$ An analysis of IVCM parameters in relation to the degree of clinically apparent conjunctival scarring in this group has been previously presented. ${ }^{6}$

\section{Biopsy samples and staining}

The eyelid was anaesthetised with an injection of 2\% lignocaine (Vital Healthcare, India) and the eye cleaned with 5\% povidone iodine. A biopsy sample was taken using a $3 \mathrm{~mm}$ trephine from the tarsal conjunctiva, $2 \mathrm{~mm}$ from the lid margin, at the junction of the medial two-third and lateral one-third of the everted lid. All samples were taken from the eye in which the IVCM had been performed. Samples were immediately placed in 10\% neutral buffered formalin and subsequently embedded in paraffin wax. Sections, $4 \mu \mathrm{m}$ thick, were cut perpendicular to the conjunctival surface and stained with H\&E, elastin/Van Gieson (EVG) and stained by immunohistochemistry for CD45 as a marker of nucleated haematopoietic cells (mouse monoclonal antibody, Dako, UK, Clones 2B11+PD7/26, $1: 800)$ and for CD83 as a marker of mature DCs (mouse monoclonal antibody, Abd Serotec, UK, Clone HB1e, $1: 75)$. Prior to immunohistochemical staining, sections were dewaxed and pressure cooked for $4 \mathrm{~min}$ in deionised water with antigen retrieval solution (Vector Lab, UK). After incubation at room temperature for $30 \mathrm{~min}$ with the primary antibody, sections were incubated with a biotinylated secondary antibody, followed by streptavadinconjugated horseradish peroxidase (Dako, UK), and finally 3,3'-diaminobenzidine (Dako, UK) was used as the chromogen. Endogenous peroxidase was inhibited using Real Endogenous Peroxidase Block (Dako, UK). The slides were counterstained with Harris haematoxylin. All immunostaining procedures were performed using the Dako autostainer (Dako, UK). Positive control samples were provided by human tonsil sections, and omission of primary antibody controls were used throughout. This study is part of a wider survey of the immunohistochemistry of these cases, and in this regard, other components of the study provide an important source of 'irrelevant' antibody controls of non-specific primary antibody staining.

\section{Microscopic examination of biopsy samples}

Grading was performed by ocular pathologists who were masked to the clinical and IVCM findings. The grading was performed on the subepithelial tissue (lamina propria and stroma unless stated otherwise) using the parameters described below and illustrated in figure 1 . These were chosen to enable comparison with the IVCM scarring score, inflammatory infiltrate and presence of DFC.

\section{Conjunctival scarring}

The degree of scarring was assessed using cross-polarised light for both the subepithelial conjunctival stroma and the tarsus. Initial comparison of the examination of picrosirius red and other tinctorial stains under cross-polarised light showed that the organisation of collagen bundles was apparent with a variety of staining methods. For assessment purposes we used EVG-stained preparations as these were available for all the samples, and we were able to identify all critical features of interest. In healthy subepithelial tissue, short connective tissue fibres running parallel to the surface in an ordered manner could be seen. In healthy tarsus, long thick bundles of collagen could be seen passing between the Meibomian glands, perpendicular to the surface, which terminated in shorter fibres running parallel to the surface, giving rise to a ' $\mathrm{T}$ ' appearance. Tissue appearing healthy was graded as 0 , and grades $1-3$ used for progressive disorder of the normal appearance, figure 1A,B.

\section{Inflammatory cells}

The inflammatory cell density was assessed using the H\&E stained slides using an ordinal scale: $0=$ scattered cells; $1=$ few cells; $2=$ moderate number of cells; $3=$ abundant $/$ confluent cells, figure $1 \mathrm{C}$.

\section{Dendritic cells}

Cellular staining with the CD83 antibody was graded as an indicator of DC presence using an ordinal scale: $0=$ no/very few cells; $1=$ occasional cells; $2=$ moderate cells; $3=$ abundant $/$ confluent cells, figure 1D. To further characterise the presence of DC we counted the number CD45 cells with dendritic morphology, where the dendritic processes $\geq$ twice the width of the nucleus (in order to minimise the inclusion of CD45 cells which are not $\mathrm{DC}$ ), figure $1 \mathrm{E}$. The field with the greatest density of cells using $\times 400$ magnification was used for counting. Cells were counted in the epithelium and subepithelial stroma.

\section{Data analysis}

Data were entered into Access 2007 (Microsoft) and analysed using STATA V.11.0 (StataCorp LP, Texas, USA). Median values are presented, as much of the data was non-normally distributed. The Kruskal-Wallis test (with a correction for ties) was used to compare the respective scarring and inflammatory cell measurements ascertained by IVCM and histology. A non-parametric test for trend was used to look at the IVCM scarring score by the histological tarsal connective tissue scarring grade. Fisher's exact tests were used to determine the strength of association between IVCM DFC and immunohistochemical CD83 staining. The Wilcoxon rank-sum test was used to test for a difference in the number of CD45 cells with a dendritic morphology on immunohistochemistry according to the presence of IVCM DFC.

\section{RESULTS}

\section{Study participants, IVCM and histopathological findings}

Demographic, IVCM and histopathological characteristics of the participants are shown in table 1. Approximately half the 

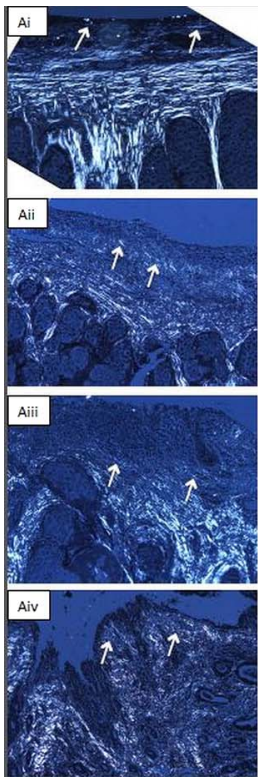
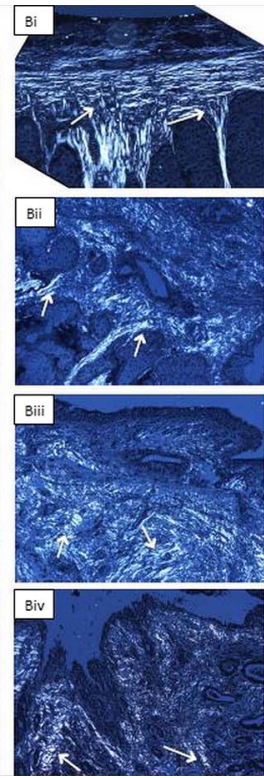
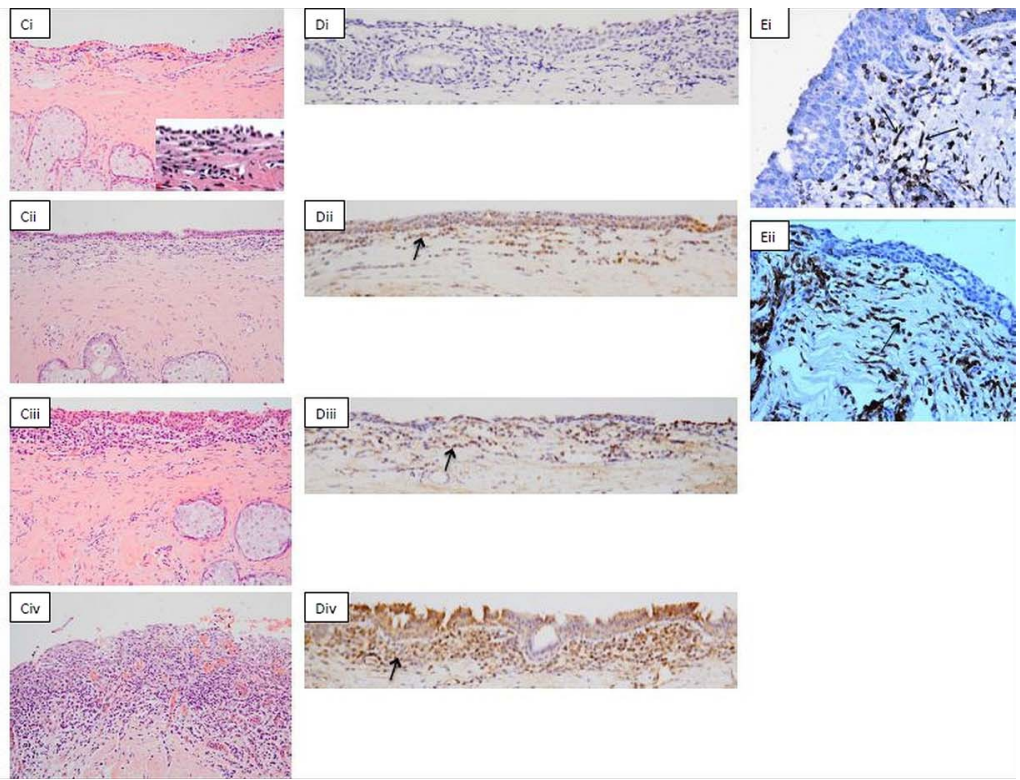

Figure 1 Example images for conjunctival histological grading. (A) Connective tissue scarring in the subepithelial space. Cross-polarised light is used. (i) Shows normal tissue (grade 0) with collagen fibres parallel to the surface (arrows). (ii-iv) Show progressive disorganisation of this appearance (grades 1-3) with the arrows indicating the subepithelial collagen fibres. Original magnification $\times 100$. (B) Connective tissue scarring in the tarsal tissue. Cross-polarised light is again used. (Bi) shows normal tissue (grade 0 ) with long collagen fibres between the meibomian glands which join shorter fibres next to the stroma forming a ' $T$ ' sign in normal tissue (arrows). (ii-iv) Show progressive disorganisation of this appearance (grades 1-3). Original magnification $\times 100$. (C) Inflammatory cell infiltrate in the subepithelium. Heamtoxylin and eosin stain used, arrows show inflammatory cells. (i-iv) Correspond to grades $0-3$. The inset in (Ci) shows the inflammatory cells at a higher magnification. Original magnification $\times 200$. (D) CD83 cell in the subepithelium. These are stained brown as shown by the arrows. (i-iv) Correspond to grades $0-3$. Original magnification $\times 200$. (E) CD45 cells with a dendritic morphology. These are also stained brown but have a dendritic morphology, as shown by the arrows. Original magnification $\times 400$.

participants were female, median age was 69 years (range 3591 years), and the most common ethnic group was Maasai (41.8\%) followed by Chagga (26.9\%). Demographic, clinical and IVCM characteristics according to the presence of trachomatous scarring have been previously presented. ${ }^{6}$ The IVCM connective tissue analysis tended to show either little scarring or advanced scarring, consistent with participants being either controls with no scarring, or cases with severe clinical scarring. DFC, defined by IVCM, were present in almost one-third $(31.5 \%)$ of the participants, and almost all of these had clinically visible scarring and inflammation. ${ }^{6}$ The immunohistological grading tended to be predominantly mild-moderate for all the parameters graded, although there was a range of grades present for each parameter.

\section{Comparison of histological and IVCM connective tissue scarring assessments}

The IVCM connective tissue scarring score was associated with histological subepithelial stromal $(\mathrm{p}=0.0008)$ and tarsal connective $(p=0.002)$ tissue scarring grades (table 2$)$. Any histological scarring in the subepithelial tissue was associated with increased IVCM scarring scores compared with no scarring. The IVCM scarring score showed a progressive increase with tarsal histological scarring grade ( $\mathrm{p}$-trend $<0.001$; see online supplementary figure S3).

\section{Comparison of histological and IVCM inflammatory cell density assessments}

There was limited evidence of an association between IVCM inflammatory cell density and histological inflammatory cell grade on $H \& E$ staining $(p=0.05$; table 3$)$.

\section{Comparison of immunohistological DC parameters and IVCM DFC}

The presence of IVCM DFC showed no association with the presence of CD83 cells (table 4). The median number of CD45 cells which had a dendritic morphology per field was similar whether or not IVCM DFC had been identified, table 4.

\section{DISCUSSION}

In this study, we found that the IVCM connective tissue scarring score was strongly associated with the histological assessment of scarring in the tarsal area. The histological quantification of scarring has itself not been well defined, particularly in the tarsal conjunctival surface. We developed a novel system for grading the amount of scarring present, using polarised light to directly visualise connective tissue fibres. Previous descriptions of conjunctival scarring in other diseases using IVCM have been very limited. ${ }^{12} 13$

The presence of small, round, hyper-reflective bodies has been widely reported in IVCM studies of the conjunctival surface, and these are generally accepted as representing the nuclei of inflammatory cells. ${ }^{14-16}$ We found limited evidence of an association between this apparent IVCM cell infiltrate and inflammatory cells seen histologically. Leucocytes are the most likely explanation for the cell type responsible for these hyperreflective bodies seen on IVCM with few plausible alternative explanations. Previous studies have found a similar IVCM appearance to be associated with clinical inflammation in vernal keratoconjunctivitis, and to show a rapid increase in rabbit conjunctiva after the injection of lipopolysaccharide. ${ }^{15}{ }^{17}$ A stronger association may have been found between the IVCM and histological inflammatory cell infiltrates in this study if the IVCM 
Table 1 Patient demographics, in vivo confocal microscopy and immunohistological findings

\begin{tabular}{|c|c|c|}
\hline & $n / 67$ & $(\%)$ \\
\hline \multicolumn{3}{|l|}{ Demographic characteristics } \\
\hline Female & 37 & $(55.2)$ \\
\hline \multicolumn{3}{|l|}{ Age (years) } \\
\hline $34-45$ & 3 & $(4.5)$ \\
\hline $45-55$ & 8 & (11.9) \\
\hline $55-65$ & 16 & $(23.9)$ \\
\hline $65-75$ & 18 & $(26.9)$ \\
\hline $75-85$ & 16 & $(23.9)$ \\
\hline $85-95$ & 6 & $(9.0)$ \\
\hline \multicolumn{3}{|l|}{ Ethnic group } \\
\hline Maasai & 28 & $(41.8)$ \\
\hline Chagga & 18 & $(26.9)$ \\
\hline Pare & 8 & $(11.9)$ \\
\hline Other & 13 & (19.4) \\
\hline \multicolumn{3}{|l|}{ In vivo confocal microscopy findings } \\
\hline Median connective tissue scarring score (IQR) $n=51^{*}$ & 1.00 & $(0.38-2.50)$ \\
\hline \multicolumn{3}{|l|}{ Connective tissue scarring grade $(n(\%)) n=51^{*}$} \\
\hline $0-1$ & 26 & $(51.0)$ \\
\hline$>1-2$ & 9 & $(17.6)$ \\
\hline$>2-3$ & 16 & $(31.4)$ \\
\hline Median inflammatory cell infiltrate (IQR) $n=50^{*}$ & 1207 & $(830-2034)$ \\
\hline Dendritiform cells present ( $(\%)) n=54^{*}$ & 17 & $(31.5)$ \\
\hline \multicolumn{3}{|l|}{ Immunohistological findings } \\
\hline \multicolumn{3}{|l|}{ Subepithelial connective tissue scarring grade $(n(\%)) n=60 \dagger$} \\
\hline 0 & 29 & $(48.3)$ \\
\hline 1 & 18 & $(30.0)$ \\
\hline 2 & 12 & $(20.0)$ \\
\hline 3 & 1 & $(1.7)$ \\
\hline \multicolumn{3}{|l|}{ Tarsal connective tissue scarring grade $(n(\%)) n=45 \dagger$} \\
\hline 0 & 31 & $(68.9)$ \\
\hline 1 & 5 & $(11.1)$ \\
\hline 2 & 7 & $(15.6)$ \\
\hline 3 & 2 & $(4.4)$ \\
\hline \multicolumn{3}{|l|}{ Inflammatory cell infiltrate on H\&E stain $(n(\%)) n=65 \dagger$} \\
\hline 0 & 16 & $(24.6)$ \\
\hline 1 & 29 & $(44.6)$ \\
\hline 2 & 11 & $(16.9)$ \\
\hline 3 & 9 & $(13.9)$ \\
\hline \multicolumn{3}{|l|}{ Cellular CD83 staining $(\mathrm{n}(\%)) \mathrm{n}=65 t$} \\
\hline 0 & 14 & $(21.5)$ \\
\hline 1 & 32 & $(49.2)$ \\
\hline 2 & 18 & $(27.7)$ \\
\hline 3 & 1 & $(1.5)$ \\
\hline $\begin{array}{l}\text { Median number of CD45 cells with dendritic morphology } \\
\text { (IQR) } n=67 t\end{array}$ & 6 & $(4-8)$ \\
\hline
\end{tabular}

* In vivo confocal microscopy was successfully performed on 54 out of the 67 participants. A small number of the scans were not suitable for connective tissue scarring grading or inflammatory cell counting.

tSome samples did not yield gradable sections and several sections did not have adequate tarsal tissue for grading of the connective tissue scarring.

had been performed in the precise area where the biopsy was taken from. Unfortunately, it is not possible to ensure that the site of the biopsy and the IVCM image are at exactly the same point, as the image area is small $(400 \times 400 \mu \mathrm{m})$, and the confocal microscope cap is relatively bulky, making it difficult to identify the precise location of the scan. Instead, mean values derived from multiple scans from all over the tarsal surface were
Table 2 Comparison of histological and in vivo confocal microscopy connective tissue scarring assessments

\begin{tabular}{llrl}
\hline & $\mathbf{n}$ & $\mathbf{p}$ Value \\
\hline Histological subepithelial & Median IVCM scarring score & & \\
scarring grade & & \\
0 & 0.50 & 21 & 0.0008 \\
1 & 2.50 & 15 & \\
2 & 2.00 & 7 & \\
3 & 2.00 & 1 & \\
Histological tarsal scarring & Median IVCM scarring score & & \\
grade & $(95 \%$ Cl) & & \\
0 & 0.47 & 26 & 0.002 \\
1 & 1.75 & 3 & \\
2 & 2.5 & 5 & \\
3 & 2.5 & 2 & \\
\hline${ }^{*}$ Kruskal-Wallis test. & & & \\
IVCM, in vivo confocal microscopy. & &
\end{tabular}

used. It is possible that the particular biopsy site may not have been representative of the rest of the tarsal conjunctiva.

We found no association between the presence of IVCM DFC and the histological identification of DC. This may be a result of study limitations. To maximise the number of histological sections available, they were cut perpendicular to the conjunctival surface. This may limit the detection of DC, as the dendritic processes appear to spread out parallel to the surface, and so may be more easily identified by IVCM, where the image obtained is parallel to the surface. IVCM images were obtained for analysis from multiple points across the tarsal conjunctiva, with summary values for scarring, and other indices being averaged from these. These were compared with a single biopsy specimen, and comparison of the two techniques may be limited. It was not possible to be certain about scanning and sampling the same site, as discussed above, again limiting the comparisons made. While the IVCM and histological grading were performed in a masked manner, it was still possible for the IVCM scan or histological tissue section itself to provide clues to the clinical status of the patient with the potential to unmask the observers leading to bias. However, every attempt was made to perform the grading as objectively as possible using clearly specified criteria.

DC immunophenotyping is complex and requires a combination of staining intensity coupled with positive and negative markers in order to confidently identify different DC types. ${ }^{18} 19$ We had insufficient tissue to exhaustively characterise this cell population and selected markers which we expected would best indicate a number of cell types including DC. The IVCM grading

Table 3 Comparison of histological and in vivo confocal microscopy inflammatory cell density assessments

\begin{tabular}{llrl}
\hline $\begin{array}{l}\text { Histological inflammatory } \\
\text { infiltrate grade }\end{array}$ & $\begin{array}{l}\text { Median IVCM inflammatory } \\
\text { cell density, cells/mm }\end{array}$ & $\mathbf{n}$ & $\mathbf{p}$ Value* \\
\hline 0 & 1242 & 15 & 0.05 \\
1 & 948 & 22 & \\
2 & 2088 & 7 & \\
3 & 1539 & 5 & \\
\hline *Kruskal-Wallis analysis of variance. & & & \\
IVCM, in vivo confocal microscopy. & &
\end{tabular}


Table 4 Comparison of immunohistological dendritic cell parameters and the presence of in vivo confocal microscopy DFC

\begin{tabular}{|c|c|c|c|c|c|}
\hline & \multicolumn{2}{|c|}{$\begin{array}{l}\text { DFC } \\
\text { present }\end{array}$} & \multicolumn{2}{|c|}{$\begin{array}{l}\text { DFC } \\
\text { absent }\end{array}$} & \multirow[b]{2}{*}{$p$ Value } \\
\hline & $\mathrm{n}$ & $\%$ & $\mathbf{n}$ & $\%$ & \\
\hline Cellular CD83 staining & $\mathrm{n}=17$ & & $n=35$ & & $0.53^{*}$ \\
\hline 0 & 3 & 17.7 & 7 & 20.0 & \\
\hline 1 & 11 & 64.7 & 17 & 48.6 & \\
\hline 2 & 3 & 17.7 & 11 & 31.4 & \\
\hline 3 & 0 & 0.0 & 0 & 0.0 & \\
\hline $\begin{array}{l}\text { Median number of CD45 cells with } \\
\text { dendritic morphology per field }\end{array}$ & $\begin{array}{c}n=17 \\
6\end{array}$ & & $\begin{array}{c}n=37 \\
7\end{array}$ & & $0.32 \dagger$ \\
\hline
\end{tabular}

*Fisher's exact test.

tWilcoxon rank-sum test.

DFC, dendritiform cells on IVCM.

identified DFC with well-developed dendritic processes, suggesting mature DC which would usually stain with CD83. ${ }^{20} 21$ The use of cellular CD45 staining and dendritic morphology should also have identified mature DC. However, to be able to determine with greater certainty whether the IVCM DFCs represent DCs would require a number of further stains.

An alternative explanation for the apparent discordance between the IVCM and histological findings is that the DFC seen on IVCM are not actually DCs. There has been no previous work on the histological characterisation of these DFCs. One possibility, suggested by other studies, is that these dendritiform structures are fibroblasts, which can have marked dendritic extensions. $^{22} 23$ IVCM studies have shown similar DFCs to be increased in microbial infection, vernal keratoconjunctivitis, contact lens wear, immune-mediated inflammation and pterygium. ${ }^{17} 2425$ Many of these conditions are associated with scarring, where fibroblasts would be expected to be present. The cornea contains numerous keratocytes, and these can transform into activated corneal fibroblasts in response to injury. ${ }^{26} 27$ The comprehensive identification of these conjunctival DFCs requires further study.

We chose to use an ordinal scale for the immunohistological grading of inflammatory cells and cellular CD83 staining, with the observer masked to the clinical status. Alternative forms of analysis include cell counts on representative fields. However, this is open to bias unless the fields selected are chosen at random, which itself can present difficulties if the infiltrate is not evenly distributed within the tissue. Additionally, the use of representative fields restricts the amount of tissue used for the assessment.

This is the first time that IVCM analysis has been objectively and quantitatively compared with immunohistological findings in the ocular surface. Masked observers and defined grading systems on a large number of participants were used, thereby allowing significance testing to be performed. These findings are likely to have relevance for other scarring diseases of the ocular surface, such as mucous membrane pemphigoid.

Acknowledgements We would like to acknowledge the help of Heidi Barnes and Caroline Thaung at the Institute of Ophthalmology with processing the biopsy samples and with using the microscope camera. We would also like to thank Anthony Hall and the theatre staff at the Eye Department of the Kilimanjaro Christian Medical Centre for their help with recruiting participants.

Contributors Conception and design: VHH, MJH, DCWM, RLB \& MJB. Acquisition of data: VHH, PM \& WM. Interpretation of IVCM images: VHH \& MJB. Histopathological processing and analysis of slides: MJH, IAC, JP \& PL. Statistical analysis: VHH \& HW. Drafting and revising the article: VHH, MJH, IAC, HAW, WM, DCWM RLB, MJB \& PL.

Funding Wellcome Trust \& British Council for Prevention of Blindness. Competing interests None.

Ethics approval London School of Hygiene and Tropical Medicine; Tanzanian National Institute of Medical Research Ethics Committee.

Provenance and peer review Not commissioned; externally peer reviewed.

Open Access This is an Open Access article distributed in accordance with the Creative Commons Attribution Non Commercial (CC BY-NC 3.0) license, which permits others to distribute, remix, adapt, build upon this work non-commercially, and license their derivative works on different terms, provided the original work is properly cited and the use is non-commercial. See: http://creativecommons.org/licenses/by-nc/3.0/

\section{REFERENCES}

1 Jalbert I, Stapleton F, Papas E, et al. In vivo confocal microscopy of the human cornea. Br J Ophthalmol 2003;87:225-36.

2 Rito C, Pineiro-Maceira J. [Reflectance confocal microscopy in the diagnosis of cutaneous melanoma]. An Bras Dermatol 2009;84:636-42.

3 Li WB, Zuo XL, Li CQ, et al. Diagnostic value of confocal laser endomicroscopy for gastric superficial cancerous lesions. Gut 2011;60:299-306.

4 Niederer RL, McGhee CN. Clinical in vivo confocal microscopy of the human cornea in health and disease. Prog Retin Eye Res 2010;29:30-58.

$5 \mathrm{Hu}$ VH, Massae P, Weiss HA, et al. In vivo confocal microscopy of trachoma in relation to normal tarsal conjunctiva. Ophthalmology 2011;118:747-54.

$6 \mathrm{Hu}$ VH, Weiss HA, Massae $\mathrm{P}$, et al. In vivo confocal microscopy in scarring trachoma. Ophthalmology 2011;118:2138-46.

7 Meltendorf C, Buhren J, Bug R, et al. Correlation between clinical in vivo confocal microscopic and ex vivo histopathologic findings of Salzmann nodular degeneration. Cornea 2006;25:734-8.

8 De Nicola R, Labbe A, Amar N, et al. [In vivo confocal microscopy and ocular surface diseases: anatomical-clinical correlations]. J Fr Ophtalmol 2005;28:691-8.

9 Dawson C, Jones B, Tarizzo M. Guide to trachoma control in programmes for the prevention of blindness. Geneva: World Health Organization, 1981.

$10 \mathrm{Hu}$ VH, Massae $\mathrm{P}$, Weiss HA, et al. Bacterial infection in scarring trachoma. Invest Ophthalmol Vis Sci 2011;52:2181-6.

$11 \mathrm{Hu}$ VH, Weiss HA, Ramadhani AM, et al. Innate immune responses and modified extracellular matrix regulation characterize bacterial infection and cellular/connective tissue changes in scarring trachoma. Infect Immun 2012;80:121-30.

12 Hu Y, Adan ES, Matsumoto Y, et al. Conjunctival in vivo confocal scanning laser microscopy in patients with atopic keratoconjunctivitis. Mol Vis 2007;13:1379-89.

13 Labbe A, Dupas B, Hamard $\mathrm{P}$, et al. In vivo confocal microscopy study of blebs after filtering surgery. Ophthalmology 2005;112:1979.

14 Zhivov A, Stachs 0, Kraak R, et al. In vivo confocal microscopy of the ocular surface. Ocul Surf 2006;4:81-93.

15 Liang $H$, Baudouin $C$, Labbe $A$, et al. In vivo confocal microscopy and ex vivo flow cytometry: new tools for assessing ocular inflammation applied to rabbit lipopolysaccharide-induced conjunctivitis. Mol Vis 2006;12:1392-402.

16 Le Q, Hong J, Zhu W, et al. In vivo laser scanning confocal microscopy of vernal keratoconjunctivitis. Clin Experiment Ophthalmol 2011;39:53-60.

17 Le Q, Hong J, Zhu W, et al. In vivo laser scanning confocal microscopy of vernal keratoconjunctivitis. Clin Experiment Ophthalmol 2011;39:53-60.

18 Baudouin C, Brignole F, Pisella PJ, et al. Immunophenotyping of human dendriform cells from the conjunctival epithelium. Curr Eye Res 1997;16:475-81.

19 Merad M, Ginhoux F, Collin M. Origin, homeostasis and function of Langerhans cells and other langerin-expressing dendritic cells. Nat Rev Immunol 2008;8:935-47.

20 Prazma CM, Tedder TF. Dendritic cell CD83: a therapeutic target or innocent bystander? Immunol Lett 2008;115:1-8.

21 Breloer M, Fleischer B. CD83 regulates lymphocyte maturation, activation and homeostasis. Trends Immunol 2008;29:186-94.

22 Rhee S, Grinnell F. P21-activated kinase 1: convergence point in PDGF- and LPAstimulated collagen matrix contraction by human fibroblasts. J Cell Biol 2006;172:423-32.

23 Abe M, Ho CH, Kamm KE, et al. Different molecular motors mediate plateletderived growth factor and lysophosphatidic acid-stimulated floating collagen matrix contraction. J Biol Chem 2003;278:47707-12.

24 Mastropasqua L, Nubile M, Lanzini M, et al. Epithelial dendritic cell distribution in normal and inflamed human cornea: in vivo confocal microscopy study. Am J Ophthalmol 2006;142:736-44.

25 Wang Y, Zhao F, Zhu W, et al. In vivo confocal microscopic evaluation of morphologic changes and dendritic cell distribution in pterygium. Am J Ophthalmol 2010;150:650-55 e1.

26 Fini ME. Keratocyte and fibroblast phenotypes in the repairing cornea. Prog Retin Eye Res 1999;18:529-51.

27 West-Mays JA, Dwivedi DJ. The keratocyte: corneal stromal cell with variable repair phenotypes. Int J Biochem Cell Biol 2006;38:1625-31. 